\title{
Estructura, correlatos y predictores del estrés por aculturación. El caso de personas refugiadas colombianas en Costa Rica*
}

\author{
Structure, correlates and predictors of acculturation stress. \\ A case study with colombian refugees in Costa Rica
}

Recibido: mayo 4 de 2010 ～Revisado: junio 15 de 2010 | Aceptado: julio 15 de 2010

\author{
KARLA UGALDE-WATSON ${ }^{* *}$ \\ VANESSA SMITH-CASTRO \\ Universidad de Costa Rica \\ MARJORIE MORENO-SALAS \\ Universidad Católica de Costa Rica \\ JoSE Miguel RODRÍGUEZ-GARCíA \\ Universidad de Costa Rica
}

RESUMEN

Cien personas refugiadas colombianas completaron un cuestionario que medía el estrés experimentado durante el proceso de adaptación a la sociedad costarricense, las redes de apoyo construidas en Costa Rica, la frecuencia con que se han visto discriminadas y su nivel de salud mental. Los datos muestran que el estrés por aculturación se estructuran en cuatro dimensiones: el estrés experimentado por los preparativos para la salida, el estrés derivado de su condición de refugiados, el estrés experimentado ante las necesidades económicas y el estrés vivido ante los retos de la adaptación cultural a la sociedad costarricense. Los preparativos para la salida del país son los eventos de mayor estrés reportado. La discriminación percibida y los años de residencia en Costa Rica aparecieron como los principales predictores de los tipos de estrés posinmigración, una vez controlados los efectos de la salud y las redes de apoyo.

Palabras clave autor

Refugiados colombianos, estrés por aculturación, percepción de la discriminación, salud mental, redes de apoyo social.

Palabras clave descriptores

Estrés, autorreporte, psicología, inmigración.

Para citar este artículo. Ugalde-Watson, K., SmithCastro, V., Moreno-Salas, M. \& Rodriguez-García, J. M. (2011). Estructura, correlatos y predictores del estrés por aculturación. El caso de personas refugiadas colombianas en Costa Rica. Universitas Psychologica, 10 (3), 759-774.

Artículo de investigación desarrollado con financiamiento del proyecto número 723-A83-19 "Discriminación Social: consecuencias psicológicas y estrategias de afrontamiento" por parte de la Vicerrectoría de Investigación de la Universidad de Costa Rica.

** Corresponding author: Vanessa Smith-Castro, Instituto de Investigaciones Psicológicas, Universidad de Costa Rica, Apdo. 11501-2060, San Pedro, San José, Costa Rica, E-mail: Vanessa.smith@ucr.ac.cr; karlauw@yahoo.com; vanessa.smith@ucr.ac.cr; maryyuri12@gmail.com; jrodriguez@sinaes.ac.cr

\section{A B S T R A C T}

One hundred colombian refugees completed a questionnaire measuring the level of stress they have experienced during the process of adaptation to the Costa Rican society; the social networks they have built in Costa Rica; the frequency with which they have been discriminated against; and their mental health. Data show four dimensions for acculturative stress: the stress produced by the arrangements to leave Colombia; the stress derived from their status as refugees; the stress derived from their socioeconomic needs; and the stress resulting from coping with the demands of the social and cultural adaptation to the host society. Participants reported significant higher levels of stress when planning the emigration from Colombia. Perceived discrimination and years of residence in Costa Rica were the principal predictors of acculturative stress in Costa Rica, above and below of the effect of other potential predictors, as social networks and mental health. Key words author

Colombian refugees, acculturative stress, perceived discrimination, mental health, social support networks.

Key words plus

Stress, self-report, psychology, immigration. 
Costa Rica es uno de los principales países receptores de civiles colombianos desplazados. El número de refugiados en Costa Rica se estima en 11.000, de los cuales el $90 \%$ son de origen colombiano (Alto Comisionado de las Naciones Unidas para los Refugiados [ACNUR], 2008). Estas personas se han visto obligadas a dejar su país de origen por temor fundado en la persecución debida a su etnia, culto religioso, nacionalidad o pertenencia a algún grupo social o político, por lo cual solicitan la protección del Estado costarricense por medio del Estatuto de Refugio.

Para las personas refugiadas la emigración generalmente representa la única vía para preservar su integridad física, pero a la vez implica la desvinculación forzosa de su país de origen, su cultura, sus amigos y familiares. Adicionalmente, las personas refugiadas se ven obligadas a invertir importantes recursos y energía en adaptarse al país de acogida.

Este proceso de adaptación psicológica y sociocultural a las sociedades receptoras se ha estudiado desde múltiples perspectivas, pero principalmente desde el enfoque de la aculturación (Berry, 2008; Berry \& Sam, 1997; Jasinskaja-Lahti, Liebkind, Jaakkola \& Reuter, 2006). La aculturación, como proceso que resulta del contacto entre dos o más grupos culturales, tiene un impacto a nivel grupal produciendo transformaciones en estructuras sociales e institucionales, y un impacto a nivel individual generando cambios actitudinales y conductuales (Berry, 2005).

A nivel grupal el estudio de la aculturación requiere de la consideración de las características claves de los colectivos. Un aspecto relevante para caracterizarlos, es la naturaleza de su reubicación. El contacto voluntario es característico de las personas que escogen reubicarse en otras naciones (migrantes y residentes); mientras que la movilidad forzosa es común en los desplazados por conflictos (refugiados y solicitantes de asilo). La investigación muestra que los grupos con altos niveles de movilidad involuntaria, comúnmente presentan mayores problemas de ajuste sociocultural (Berry, 1997).

A nivel individual es pertinente considerar los cambios psicológicos que las personas experimentan en los grupos y su eventual adaptación a las nuevas situaciones en la reubicación (Graves, 1967). Estudios previos muestran una gran variabilidad en las experiencias individuales de aculturación; dichos cambios se pueden expresar como ligeras modificaciones conductuales (cambios en las formas de vestir, hablar, comer, etc.) o bien como transformaciones profundas y problemáticas que se manifiestan en forma de incertidumbre, ansiedad o depresión (Berry, 2005). El término estrés por aculturación se ha acuñado precisamente para describir este tipo de situaciones, en donde las demandas de adaptación a la nueva cultura exceden las capacidades de las personas para afrontarlas (Williams \& Berry, 1991).

La investigación ha documentado una amplia gama de predictores de este tipo de estrés en distintos niveles de análisis. A nivel individual se destacan las características sociodemográficas de las personas, como la cantidad de tiempo de residir en el país (Miranda \& Mathery, 2000) y su estatus socioeconómico (Hovey, 2000; Jasinskaja-Lahti et al., 2006). Por otro lado, se señalan variables psicosociales como el grado de identificación con su grupo de referencia y prácticas como el uso del lenguaje nativo (Ward, 2006). También sobresalen aspectos de personalidad como la autoeficacia, los estilos de afrontamiento y el estado general de salud mental y física (Knipscheer \& Kleber, 2006; Liebkind, 1996; Nesdale, Dharmalingam \& Kerr, 1987; Ward \& Kennedy, 1993; Williams \& Berry, 1991). A nivel contextual, el contacto positivo con redes de apoyo social y comunal y su contraparte, la discriminación y rechazo por parte de la comunidad receptora, se documentan como dos importantes predictores del grado en que el proceso de aculturación se vive como estresante o no (Berry, 2005; Keel \& Drew, 2004).

La presente investigación tiene dos objetivos: en primer lugar, nos interesa caracterizar el estrés por aculturación en personas refugiadas colombianas en Costa Rica y, en segundo lugar, analizar el impacto relativo de la discriminación, las redes de apoyo y la salud mental, sobre este tipo de estrés. Investigaciones previas han mostrado que estos factores pueden ser decisivos para la adaptación de estos grupos poblacionales en las sociedades 
receptoras. El estudio sistemático de estas variables podría contribuir al desarrollo de programas de intervención que aseguren su plena integración.

\section{Discriminación social}

La discriminación, en tanto dimensión conductual del prejuicio, se refiere al tratamiento diferencial, por lo general injusto, del que es objeto una persona en sus interacciones cotidianas, por el simple hecho de pertenecer a una categoría social específica (Allport, 1954).

La discriminación tiene importantes efectos psicológicos, porque implica reconocer las acciones de los otros no solo como ilegítimas, sino también como reacciones negativas ante una categoría social de pertenencia altamente apreciada por los individuos; ya que la "razón" misma de la discriminación, el propio grupo de referencia, es una fuente fundamental de definición y valoración personal (sobre las implicaciones de pertenecer a categorías sociales estigmatizadas, véanse Tajfel \& Turner, 1979). La investigación empírica muestra que la discriminación afecta negativamente la calidad de vida de las personas en diversos niveles y se ha encontrado negativamente asociada a la salud física y mental (Knipscheer \& Kleber, 2006; Smith, 2005; Zlobina, Basabe, Paez \& Furnham, 2005).

Aunque Costa Rica es un país pluricultural y la condición de refugio supone el respeto de los derechos de igualdad y no discriminación garantizados por la constitución política, las relaciones interculturales costarricenses están cargadas de la discriminación estructural, el etnocentrismo y la ideología racista residual del colonialismo europeo (Smith, 2006). Muchas personas refugiadas colombianas son percibidas bajo estigmas que afectan su proceso de integración local. Investigaciones previas indican que las personas refugiadas en Costa Rica, han sido víctimas de comentarios negativos por parte de costarricenses en tanto "traficantes de drogas", "usurpadores del trabajo de los nacionales" e "indeseables por ser extranjeros", entre otros (UCR \& ACNUR, 2004). Estos estudios indican además que los principales padecimientos señalados por las personas colombianas refugiadas, son la tristeza, el estrés, la depresión, el dolor de cabeza, el insomnio, los problemas digestivos o el nerviosismo.

Para efectos de la presente investigación, medimos la experiencia subjetiva del trato discriminatorio o discriminación percibida, sin acudir a criterios objetivos de discriminación. Es, precisamente, esta experiencia subjetiva de trato injusto basado en la categorización social la que pone en marcha una serie de mecanismos cognitivos y afectivos que permiten al sujeto afrontar la discriminación. Al mismo tiempo, se trata de una experiencia vital que puede comprometer su adaptación psicológica y sociocultural.

\section{Redes de apoyo}

Según Ye (2005), el apoyo social es un tipo de comunicación verbal y no verbal implantado en la estructura de las relaciones sociales que se presenta entre receptores y proveedores, permitiendo poner bajo control una situación de incertidumbre. Jasinskaja-Lahti et al. (2006) lo entienden como el contacto social activo con elementos de soporte tales como las amistades y la familia.

Además de la asistencia tangible, las formas primarias del apoyo social incluyen el soporte informativo y emocional. El apoyo informativo es la provisión de asesoría o guía relevante que puede incrementar las percepciones de control sobre la propia situación y, consecuentemente, facilitar el manejo del estrés por aculturación y el afrontamiento de situaciones difíciles. El apoyo emocional está asociado con las expresiones verbales y no verbales de interés o preocupación, que se transmiten por mensajes que usualmente fortalecen la autoestima y la sensación de que otras personas comprenden o acompañan al individuo (Ye, 2005). En general, la investigación muestra que las redes de apoyo social tienen un efecto positivo en el bienestar general individual y en el ajuste sociocultural, ya que posibilitan un afrontamiento más efectivo del estrés por aculturación (Jasinskaja-Lahti et al., 2006).

Tomando en cuenta la naturaleza de la movilidad forzosa de las personas refugiadas, en el presente estudio se recolectó información sobre 
diversos tipos de redes de apoyo, incluyendo la familia y los amigos que residen tanto en el país de acogida como en el país de origen, las personas de la nación anfitriona, los grupos de extranjeros residentes en el país y las instituciones sociales especializadas en acompañamiento de personas refugiadas (Jasinskaja-Lahti et al., 2006).

\section{Salud Mental}

La Organización Mundial de la Salud (2004) define la salud mental como un estado de bienestar en el cual las personas están concientes de sus propias capacidades, pueden afrontar las presiones normales de su vida cotidiana, son capaces de trabajar de forma productiva y fructífera y logran hacer un aporte a su comunidad. La investigación muestra que en le caso particular de personas obligadas a migrar, su estado de salud mental resulta ser un importante predictor del éxito de su ajuste al país de acogida, debido a que en muchos casos las razones de la emigración están vinculadas con condiciones y situaciones de violencia extrema responsables de manifestaciones propias del Síndrome de Estrés Postraumático (Berry, 1997).

Para los fines operacionales de la presente investigación, los autores se centraron en el estado reciente de bienestar mental con relación a tres áreas: ansiedad, insomnio y depresión (Knipscheer \& Kleber, 2006). Siguiendo la Clasificación Internacional de Enfermedades y Problemas Relacionados con la Salud [CIE-10] (Organización Panamericana de la Salud, 1996), se definió la ansiedad generalizada como la presencia de preocupaciones de carácter excesivo y persistente; el insomnio (no orgánico), como la dificultad para iniciar o mantener el sueño, o la sensación de no haber tenido un sueño reparador y la depresión, como la presencia de manifestaciones como el humor depresivo, la pérdida de la capacidad de interés y disfrute, la disminución de la vitalidad y el cansancio exagerado que aparece incluso tras un esfuerzo mínimo.

En síntesis, los antecedentes teóricos y empíricos sugieren que el estrés por aculturación en personas refugiadas, depende, en gran medida, de la forma en que estas son recibidas por la sociedad de acogida y de las posibilidades de desarrollar redes de apoyo en los nuevos contextos culturales. $\mathrm{Al}$ mismo tiempo, la investigación indica que el éxito de su adaptación depende de sus capacidades y potenciales individuales, las cuales pueden estar comprometidas en virtud de las circunstancias de su movilidad. Evidentemente, la relación entre estas variables es muy compleja y depende del tipo particular de los colectivos sociales y las biografías particulares, pero la investigación previa parece sugerir que: $\mathrm{H}_{1}$ ) a mayor percepción de la discriminación mayor nivel de estrés por aculturación; $\mathrm{H}_{2}$ ) a mayor nivel de salud mental menor grado de estrés por aculturación y $\mathrm{H}_{3}$ ) a mayor nivel de redes de apoyo menor grado de estrés por aculturación. En la siguiente sección presentamos los procedimientos para contrastar estas hipótesis.

\section{Método}

\section{Participantes}

En este estudio participaron 100 personas refugiadas de origen colombiano ( $57 \%$ mujeres) entre los 18 y los 68 años de edad $(M=39.68$ años, $D T=$ 11.93 años) con alrededor de 4 años de residir en Costa Rica $(M=4.14, D T=1.30)$. La mayoría de las personas residen en San José/CR (77 \%), viven en pareja (70\%) y tienen hijos(as) (76\%). Una parte importante tiene empleo remunerado (67\%), generalmente de tipo independiente (microempresas, comercio, artesanía). Poseen un nivel educativo alto, ya que un $60 \%$ cuenta con estudios técnicos o universitarios. Las principales razones de salida de Colombia fueron las amenazas/persecución (43\%), la inseguridad/violencia (31\%) y los problemas sociales/económicos (14\%), entre otros (12\%).

\section{Instrumentos}

Se diseñó un cuestionario autoaplicado que contaba mediciones de estrés por aculturación percibido, discriminación percibida, redes de apoyo, salud mental y datos sociodemográficos. 


\section{Estrés por aculturación}

Se utilizaron 33 reactivos especialmente diseñados en esta investigación que medían el grado de estrés (tensión o angustia) que sintieron los participantes en las distintas fases o aspectos del proceso de su migración, desde la decisión de salir de Colombia hasta la llegada a Costa Rica. La escala se presenta en el Apéndice. Para cada reactivo, los participantes contaban con una escala Likert de 5 puntos, de 1 (nada estresante) a 5 (muy estresante). Debido al interés de explorar la estructura del estrés por aculturación, los resultados del análisis de las características psicométricas de esta medición, se presentan posteriormente.

\section{Percepción de la discriminación}

Sobre la base de la literatura previa (Smith, 2005), se desarrollaron 18 reactivos que evalúan la frecuencia con la que los participantes han vivido experiencias de trato injusto basado en su nacionalidad. Ejemplos de los reactivos son: "iCon qué frecuencia le han negado la entrada a una fuente de soda, una discoteca o restaurante debido a su nacionalidad?" y "iCon qué frecuencia a usted le han negado un aumento, una oportunidad laboral o un mejor puesto de trabajo debido a su nacionalidad?". Los participantes contaban con una escala Likert de 5 puntos, de 1 (nunca) a 5 (siempre) para responder a los reactivos. Los ítems presentaron una estructura unidimensional, (primer valor característico $=7.53$, varianza explicada $=42 \%$ ) y mostraron cargas factoriales en el primer factor superiores a 0.39. La escala mostró un índice de consistencia interna (alfa de Cronbach) de 0.91 y las correlaciones ítem-total fueron mayores a 0.34. Debido al formato de respuesta, la escala tiene un rango de 1 a 5 , en donde altas puntuaciones indican un alto grado de exposición a experiencias discriminatorias.

\section{Salud}

Para medir el estado de salud mental se utilizaron 10 reactivos de la lista de síntomas de Hopkins en la versión española de Álvaro, Torregrosa y Garrido (1992). Ejemplos de los síntomas listados son "miedo súbito o pánico sin motivo", "mareos o desmayos" y "sentir que la vida es dura y conflictiva”. La presencia de los síntomas se reporta mediante una escala Likert de 4 puntos, de 1 (ningún problema) a 4 (muchos problemas). Los 10 reactivos se agrupan en una sola dimensión general de salud mental (primer valor característico $=$ 6.44, varianza explicada de $64.43 \%$ ), con cargas factoriales superiores a 0.60. El índice presentó una alta consistencia interna con un alfa de Cronbach de 0.94, y correlaciones ítem-total superiores a 0.55. Las respuestas a los reactivos fueron recodificadas antes de ser promediadas con el fin de que las altas puntuaciones indicaran altos niveles de salud.

\section{Redes de Apoyo}

Se solicitó a los participantes indicar la cantidad redes de apoyo (ej. familia, amigos, vecinos, instituciones, etc.) con que cuentan en Costa Rica y el tipo de apoyos que han recibido de las mismas (ej. económico, espiritual, etc.). A partir de la cantidad de redes mencionadas ( $\min .=0$, máx. =9), se construyó un índice en donde altas puntuaciones indican una mayor cantidad de redes de apoyo disponibles.

\section{Variables sociodemográficas}

Se construyeron preguntas directas sobre género, edad, estado civil, número de hijos(as), nivel educativo, zona de residencia, tiempo de residir en el país y razones de salida.

\section{Procedimiento}

Una versión preliminar de los instrumentos fue revisada por jueces expertos en materia de investigación intercultural. Posteriormente, se llevaron a cabo pruebas cognitivas del cuestionario con cuatro personas refugiadas ( 2 hombres y 2 mujeres) para detectar y solventar problemas en la comprensión de los instrumentos (Willis, 2005). 
El contacto se llevó a cabo por medio de la Agencia de Consultores y Asesores Internacionales (ACAI), agencia implementadora de los proyectos de ACNUR en Costa Rica. Los participantes fueron entrevistados en las instalaciones de ACAI o fueron visitados en sus casas. Todos los cuestionarios contenían en su introducción el consentimiento informado. Los participantes expresaron su acuerdo a participar llenando el cuestionario en su totalidad. Llenar el cuestionario tomó aproximadamente 40 minutos.

\section{Resultados}

\section{Dimensiones del estrés por aculturación}

Para estudiar las dimensiones de los reactivos sobre estrés por aculturación se llevaron a cabo análisis exploratorios de factores, utilizando el método de extracción de componentes principales y el método de rotación varimax. Los resultados se presentan en la Tabla 1. Los análisis indicaron que la mejor solución para describir la estructura subyacente a los 33 reactivos era una estructura de cuatro dimensiones: a) el estrés experimentado ante los retos de la adaptación cultural y social a la sociedad costarricense, b) el estrés derivado de su condición de refugiados, c) el estrés experimentado ante las necesidades económicas y d) el estrés experimentado por los preparativos para salir de Colombia. Estos cuatro factores explicaron un $60 \%$ de la varianza de los reactivos.

Adaptación a la sociedad receptora. El primer factor obtuvo un valor característico de 6.58 y explicó el $19.93 \%$ de la varianza total de los reactivos.

TABLA 1

Valor característico, varianza explicada y cargas factoriales de los componentes del estrés por aculturación

\begin{tabular}{|c|c|c|c|c|}
\hline & \multicolumn{4}{|c|}{ Componentes } \\
\hline & 1 & 2 & 3 & 4 \\
\hline Valor característico & 6.58 & 4.84 & 4.28 & 4.22 \\
\hline Varianza explicada & $19.93 \%$ & $14.67 \%$ & $12.99 \%$ & $12.78 \%$ \\
\hline \multicolumn{5}{|l|}{ Reactivos } \\
\hline Las actitudes de los costarricenses & 0.90 & & & \\
\hline Los comportamientos de los costarricenses & 0.85 & & & \\
\hline La mentalidad del costarricense & 0.84 & & & \\
\hline La forma de ser del costarricense & 0.82 & & & \\
\hline Los valores costarricenses & 0.72 & & & \\
\hline Iniciar el contacto con los vecinos & 0.71 & & & \\
\hline Las comidas costarricenses & 0.67 & & & \\
\hline Las costumbres costarricenses & 0.66 & 0.33 & & \\
\hline Hacer amigos & 0.65 & & & \\
\hline Establecer relaciones con las personas del trabajo & 0.54 & & 0.44 & \\
\hline Hacer los trámites para el refugio & & 0.81 & & \\
\hline Esperar el resultado de la solicitud de refugio & & 0.80 & & \\
\hline Tener que renovar el carné de refugio periódicamente & & 0.73 & & \\
\hline
\end{tabular}




\begin{tabular}{|c|c|c|c|c|}
\hline & \multicolumn{4}{|c|}{ Componentes } \\
\hline & 1 & 2 & 3 & 4 \\
\hline Salir a conocer las ciudades (Ej. paradas de buses) & 0.34 & 0.67 & 0.33 & \\
\hline Llegar a Costa Rica & & 0.58 & & 0.45 \\
\hline Realizar trámites en instituciones públicas & 0.36 & 0.58 & & \\
\hline Adaptarse a las formas de hablar de los costarricenses. & 0.46 & 0.57 & & \\
\hline La supervisión que se hace sobre las personas refugiadas & & 0.56 & 0.35 & \\
\hline Acceso de los hijos a la educación & & 0.56 & 0.36 & \\
\hline Cubrir sus necesidades básicas & & & 0.90 & \\
\hline Estabilizarse económicamente & & & 0.83 & \\
\hline Encontrar un trabajo & & & 0.71 & \\
\hline Encontrar un lugar para vivir & & & 0.64 & \\
\hline Ir de compras & 0.32 & 0.45 & 0.51 & \\
\hline Realizar trámites en bancos & & & 0.49 & \\
\hline Salir de Colombia & & & & 0.86 \\
\hline Trasladarse de Colombia a Costa Rica & & & & 0.86 \\
\hline Tomar la decisión de salir de Colombia & & & & 0.85 \\
\hline Prepararse para salir de Colombia & & & 0.49 & 0.67 \\
\hline Tener que separarse de su familia & & & & 0.60 \\
\hline Conseguir el dinero para venirse & & & & 0.54 \\
\hline Dejar de ver a los amigos & & & & 0.48 \\
\hline
\end{tabular}

Nota. Método de extracción = Componentes principales, Método de rotación = varimax con normalización Kaiser. La rotación convergió en 5 iteraciones. Factor 1 = Adaptación a la sociedad receptora, Factor 2 = Estatuto de refugio, Factor 3 = preocupaciones socioeconómicas, Factor 4 = Preparación y salida de Colombia. Solo se presentan cargas factoriales mayores a 0.30 .

Fuente: elaboración propia.

En este factor se agruparon los ítems que reflejan el estrés que se puede experimentar al entrar en contacto con las costumbres y estilos de vida de la sociedad receptora ("la mentalidad del costarricense", "las costumbres costarricenses"). Los reactivos que forman esta dimensión presentaron saturaciones factoriales mayores a 0.54 .

Adaptación al estatuto de refugio. El segundo factor agrupó aquellos reactivos referidos al estrés experimentado por la condición de refugio ("Esperar el resultado de la solicitud de refugio", "Tener que renovar el carné de refugio periódicamente").
Este factor presentó un valor característico de 4.84, explicando un $14.67 \%$ de la varianza total. Las cargas factoriales de los ítems fueron mayores a 0.56 .

Preocupaciones socioeconómicas. Este factor contenía aquellos reactivos referidos a las preocupaciones y angustias de tipo económico y social ("Encontrar un trabajo", "Estabilizarse económicamente"). El valor característico de este factor fue de 4.28 y explicó un $12.99 \%$ de la varianza. Los reactivos presentaron saturaciones mayores a 0.49 . 
Preparación para emigración. La cuarta dimensión abarcó los aspectos estresantes que se experimentaron antes de la salida ("Prepararse para salir de Colombia", "tener que separarse de la familia"). Este factor presentó un valor característico de 4.22 y explicó un $12.78 \%$ de la varianza de los reactivos, los cuales presentaron saturaciones factoriales superiores 0.48 .

A partir de estos resultados se procedió a calcular la consistencia interna de cada una de estas dimensiones. Las subescalas obtuvieron índices de consistencia interna, alfa de Cronbach, bastante adecuados (adaptación a la sociedad receptora $a=0.91$, adaptación al estatuto de refugio $\alpha=$ 0.83, preocupaciones socioeconómicas $a=0.81$, preparación para la emigración $a=0.77$ ). En todos los casos, las correlaciones ítem-total fueron superiores a 0.30 .

\section{Estrés por aculturación y las características sociodemográficas de los y las participantes}

En preparación a los análisis principales se comparó el comportamiento de las dimensiones del estrés por aculturación en el total de la muestra y se estimó su potencial asociación con el sexo, la edad y los años de residencia en el país. Los resultados de estos análisis se presentan en la Tabla 2.

Un análisis de varianza simple intrasujetos indicó que el grado de estrés experimentado dependía significativamente del ámbito o faceta del proceso de aculturación $F_{(3,297)}=133.18, p<0.001, \eta^{2}=$ 0.57. La prueba LSD indicó que los participantes reportaron haber experimentado más estrés por la preparación de la salida $(\mathrm{ME}=4.15, \mathrm{DT}=0.87)$ que por los factores socioeconómicos $(\mathrm{ME}=3.64$, $\mathrm{DT}=0.94)$, su condición de refugiados(as) $(\mathrm{ME}=$ $3.25, \mathrm{DT}=0.93)$ y las demandas de adaptación a

TABLA 2

Estadísticos descriptivos, coeficientes de consistencia interna y correlaciones bivariadas entre las variables en estudio

\begin{tabular}{|c|c|c|c|c|c|c|c|c|c|c|c|c|c|}
\hline Variables & $\mathrm{ME}$ & $D E$ & A & 1 & 2 & 3 & 4 & 5 & 6 & 7 & 8 & 9 & 10 \\
\hline $\begin{array}{l}\text { Preparación para la } \\
\text { emigración }\end{array}$ & $4.15^{\mathrm{a}}$ & 0.87 & 0.77 & 1 & $0.26^{* *}$ & $0.41 * *$ & $0.20 *$ & $0.24 *$ & -0.15 & -0.13 & -0.05 & 0.04 & 0.02 \\
\hline $\begin{array}{l}\text { Preocupaciones } \\
\text { económicas }\end{array}$ & $3.64^{\mathrm{b}}$ & 0.94 & 0.83 & & 1 & $0.47 * *$ & $0.48 * *$ & $0.31 * *$ & -0.07 & $-0.20 *$ & -0.11 & 0.07 & 0.08 \\
\hline $\begin{array}{l}\text { Adaptación al esta- } \\
\text { tuto de refugiado }\end{array}$ & $3.25^{\mathrm{c}}$ & 0.93 & 0.81 & & & 1 & $0.22 *$ & $0.41 * *$ & -0.14 & $-0.22 *$ & 0.09 & 0.15 & 0.17 \\
\hline $\begin{array}{l}\text { Adaptación a la so- } \\
\text { ciedad receptora }\end{array}$ & $2.08^{\mathrm{d}}$ & 1.01 & 0.91 & & & & 1 & $0.56^{* *}$ & $-0.21 *$ & $-0.21 *$ & -0.13 & $-0.23 *$ & 0.03 \\
\hline $\begin{array}{l}\text { Percepción de dis- } \\
\text { criminación }\end{array}$ & 1.65 & 0.55 & 0.91 & & & & & 1 & $-0.53 * *$ & $-0.22 *$ & -0.02 & -0.17 & 0.11 \\
\hline Salud Mental & 1.98 & 0.83 & 0.94 & & & & & & 1 & 0.17 & 0.04 & 0.18 & -0.04 \\
\hline Redes de apoyo & 2.09 & 1.49 & - & & & & & & & 1 & 0.08 & -0.09 & -0.18 \\
\hline Edad & 39.68 & 11.93 & - & & & & & & & & 1 & $0.24 *$ & 0.02 \\
\hline $\begin{array}{l}\text { Años de residir en } \\
\text { Costa Rica }\end{array}$ & 4.14 & 1.30 & - & & & & & & & & & 1 & -0.08 \\
\hline $\begin{array}{l}\text { Sexo: } 0=\text { mujer, } 1 \\
=\text { hombre }\end{array}$ & - & - & - & & & & & & & & & & 1 \\
\hline
\end{tabular}

Notas. $* * p<0.01, * p<0.05$. Los promedios con diferente superíndice son significativamente diferentes al $p<0.001$ en la prueba post hoc LSD. $F_{(3,297)} 133,18, p=0.001, \eta^{2}=0.16$.

Fuente: elaboración propia. 
la cultura costarricense $(\mathrm{ME}=2.08, \mathrm{DT}=1.01)$, respectivamente (todas las $p<0.001$ ).

Por otro lado, no se observaron correlaciones significativas entre las dimensiones del estrés por aculturación y el sexo, la edad o los años de residir en el país (todas las $r<|0.18|$, n.s.), con excepción del estrés experimentado por adaptarse a las costumbres de la sociedad receptora. Específicamente, se encontró una correlación negativa entre los años de residir en el país y el estrés provocado por la adaptación a la cultura costarricense $(r=$ $-0.23, p<0.05)$. Entre más años tenían los y las refugiadas de vivir en Costa Rica, menor fue el estrés que reportaron ante las demandas de adaptación a las costumbres y mentalidad costarricenses. En vista de estos resultados, los años de residir se incorporaron como predictores en los análisis de regresión para el caso específico de la predicción del estrés de adaptación a la sociedad receptora, no así en los demás modelos de regresión.

\section{El papel de la percepción de la} discriminación, la salud mental y las redes de apoyo en la predicción del estrés por aculturación

Para estimar el impacto relativo de la discriminación percibida, la salud mental y las redes de apoyo en las distintas dimensiones del estrés por aculturación, se llevaron a cabo cuatro análisis de regresión múltiple, uno para cada una de las cuatro dimensiones de estrés por aculturación. Previamente, se estimaron las correlaciones simples entre las variables. Los resultados se presentan en las Tablas 2 y 3.

En términos de los criterios, se observaron correlaciones positivas y significativas entre las diferentes dimensiones del estrés por aculturación $\left(r_{s}\right.$ entre 0.20 y $\left.0.48, p_{s}<0.05\right)$. Las personas que reportaron mayores niveles de estrés en una dimensión, también reportaron niveles superiores de estrés en las otras. En términos de las relaciones entre los predictores, las experiencias de discriminación se encontraron negativamente asociadas a la salud mental $(r=-0.53, p<0.001)$ y a las redes de apoyo $(r=-0.22, p<0.05)$. Aquellas personas que reportaron mayores experiencias de discriminación, indicaron poseer menores redes de apoyo y presentaron niveles menores de salud mental que aquellas personas que han experimento menos discriminación.

En términos de la relación entre predictores y criterios, la percepción de discriminación se encontró positivamente asociada al estrés por el estatuto de refugio $(r=0.31, p<0.001)$, los factores socioeconómicos $(r=0.41, p<0.001)$, la adaptación a la sociedad receptora $(r=0.56, p<0.001)$ y la preparación para la salida de Colombia $(r=$

TABLA 3

Análisis de regresión múltiple para las dimensiones de estrés por aculturación

\begin{tabular}{|c|c|c|c|c|c|c|c|c|}
\hline \multirow[b]{3}{*}{ Predictores } & \multicolumn{8}{|c|}{ Criterios } \\
\hline & \multicolumn{2}{|c|}{$\begin{array}{c}\text { Preparación para la } \\
\text { emigración }^{\text {a }}\end{array}$} & \multicolumn{2}{|c|}{$\begin{array}{l}\text { Adaptación al } \\
\text { estatuto de refugio }\end{array}$} & \multicolumn{2}{|c|}{$\begin{array}{c}\text { Preocupaciones } \\
\text { Socioeconómicas }^{\mathrm{c}}\end{array}$} & \multicolumn{2}{|c|}{$\begin{array}{c}\text { Adaptación a la } \\
\text { sociedad receptora }\end{array}$} \\
\hline & $\beta$ & $\mathrm{T}$ & B & $t$ & $\beta$ & $T$ & B & $t$ \\
\hline Discriminación & 0.21 & 1.77 & 0.35 & $3.08 * *$ & 0.44 & $4.01 * *$ & 0.61 & $6.30 * *$ \\
\hline Salud & -0.03 & -0.27 & 0.15 & 1.20 & 0.12 & 1.11 & 0.15 & 1.53 \\
\hline Redes de apoyo & -0.08 & -0.77 & -0.16 & -1.53 & -0.15 & -1.54 & -0.12 & -1.46 \\
\hline Años de residir en el país & - & - & - & - & - & - & -0.17 & $-1.99 *$ \\
\hline
\end{tabular}

Nota. ${ }^{*}{ }^{2} \mathrm{p}<0.01,{ }^{*} \mathrm{p}<0.05 .{ }^{a} \mathrm{R}=0.26, R^{2}=0.07$ y $\mathrm{F}=2.24$, n.s. ${ }^{b} \mathrm{R}=0.36, R^{2}=0.13$ y $\mathrm{F}=4.72, p<0.001 .{ }^{c} R=0.44, R^{2}=$ 0.19 y $F=7.68, p<0.001 .{ }^{d} R=0.63, R^{2}=0.39$ y $F=15.09, p<0.001$

Fuente: elaboración propia. 
$0.24, p<0.05)$. En concordancia con las hipótesis de investigación, los datos indican que a mayor percepción de la discriminación, mayor es el grado de estrés por aculturación, particularmente en los ámbitos vinculados a la adaptación a la sociedad receptora.

La salud mental se encontró significativamente asociada al estrés por adaptación a la sociedad receptora $(r=-0.21, p<0.05)$. Los participantes que reportaron menores niveles de salud mental, tendieron a presentar mayores niveles de estrés para adaptarse a la sociedad costarricense que los participantes que presentaron mejores niveles de salud mental. Estos datos apoyan las hipótesis de investigación, pero se limitan al estrés específicamente experimentado por las demandas de adaptación sociocultural.

Por su parte, las redes de apoyo se encontraron significativamente correlacionadas con el estrés por el estatuto de refugio $(r=-0.20, p<0.05)$, el estrés por los factores económicos $(r=-0.22$, $p<0.05)$ y el estrés por adaptarse a la sociedad receptora $(r=-0.21, p<0.05)$. Las personas que disponían de más redes reportaron un menor estrés por adaptación a la sociedad de acogida que las personas que disponían de menos redes, apoyando de manera parcial las hipótesis del estudio.

Los resultados de análisis de regresión múltiple para predecir el estrés ante la salida de Colombia, indicaron que ninguna de las variables en estudio permitía explicar la variabilidad de este tipo de estrés. La correlación múltiple entre la combinación lineal de los predictores y este criterio fue de 0.26 , en un modelo no significativo $F(3.96)=2.24$, n.s. que explicó apenas el $7 \%$ de la varianza. El modelo resultó entonces inapropiado para predecir las puntuaciones del estrés por la preparación para abandonar el país.

En el caso del estrés derivado de la condición de refugio, los resultados indicaron una asociación significativa con la combinación lineal de predictores $(R=0.36)$, en un modelo significativo $F(3.96)=4.72, p<0.001$, que explicó un $13 \%$ de la varianza del criterio. De los predictores, solo la discriminación percibida resultó significativa ( $\beta$ $=0.35, p<0.001)$. Los participantes que habían vivido más eventos de discriminación, reportaron un mayor nivel de estrés derivado de su estatuto de refugio que los participantes que reportaron menos experiencias de discriminación, manteniendo constantes su nivel de salud y sus redes apoyo.

De manera similar, los resultados para el estrés derivado de las preocupaciones socioeconómicas indicaron que este tipo de estrés se asocia con los predictores $(R=0.44)$, en un modelo significativo $F(3.96)=7.68, p<0.001$, que explicó un 19\% de su varianza. Nuevamente, solo la discriminación percibida resultó significativa $(\beta=0.44, p<$ 0.001). Los participantes que habían vivido más eventos de discriminación, reportaron un mayor nivel de estrés por su situación socioeconómica que los participantes que reportaron menos experiencias de discriminación, más allá de lo que puedan explicar su salud mental y sus redes apoyo.

El estrés ante la adaptación a la cultura costarricense se encontró altamente asociado a los predictores que incluían, esta vez, los años de residir en el país $(R=0.63)$. Se trató de un modelo altamente consistente $F(3.96)=15.09, p<$ 0.001 , que explicó aproximadamente el $40 \%$ de la varianza de este tipo de estrés. Aquí, los predictores significativos fueron la discriminación percibida en primer lugar $(\beta=0.61, p<0.001)$ y los años de residir en el país en un segundo término $(\beta=-0.17$, $p<0.05)$. Los participantes que había vivido más discriminación percibida y tenían menos años de residir en el país reportaron experimentar mayor estrés por la adaptación a la cultura costarricense que quienes habían vivido más años en el país y habían experimentado menos discriminación.

En síntesis, una vez controlados los efectos de otras variables relevantes como la salud, las redes de apoyo y los años de residir en el país, la discriminación percibida se perfiló como la principal variable que explicó la varianza del estrés por el estatuto de refugio, el estrés por las presiones socioeconómicas y el estrés por la adaptación a la sociedad receptora. Las personas que habían experimentado mayor discriminación, reportaron sentirse más estresadas por estos factores que las personas que habían vivido menos discriminación. 
Por el contrario, el estrés derivado por los procesos propios de la salida de Colombia, no estaba asociado a ninguno de los predictores estudiados.

\section{Discusión}

El objetivo de la presente investigación ha sido contribuir a la comprensión del estrés por aculturación en personas refugiadas colombianas en Costa Rica, considerando el impacto relativo de la percepción de la discriminación, las redes de apoyo y la salud mental.

Los datos indican que el estrés por aculturación está muy lejos de ser un constructo unidimensional. Se trata más bien de un fenómeno diferenciado y complejo. En este caso en particular, se detectaron cuatro ámbitos o facetas del estrés por aculturación: a) el estrés experimentado por los preparativos para la salida de Colombia, b) el estrés derivado de la condición de refugio, c) el estrés experimentado ante las necesidades económicas y d) el estrés vivido ante los retos que plantea la adaptación cultural a la sociedad costarricense.

La preparación para la salida y emigración de Colombia es la faceta de la aculturación que mayor estrés produce. Según varios autores (Hovey, 2000; Keel \& Drew, 2004; Liebkind, 1996), los eventos previos a la llegada al país se encuentran estrechamente relacionados con altos niveles de estrés por aculturación, tales como los que se documentaron en la presente investigación: abandonar el país y la cultura de origen, prepararse para la salida, separarse de la familia, asegurarse los medios económicos para abandonar el país y, principalmente, lidiar con las amenazas a la integridad física propia y/o de los seres queridos.

El segundo aspecto de mayor preocupación fueron los factores económicos. Estos resultados concuerdan con la investigación previa que indica que escasas posibilidades de contar con un empleo y las dificultades económicas se asocian con una mayor presencia de estrés por aculturación (Hovey, 2000; Jasinskaja-Lahti et al., 2006; Knipscheer \& Kleber, 2006). La movilidad involuntaria no siempre permite una adecuada preparación o planeamiento sobre la vida en el nuevo país, por lo que es usual que las personas lleguen con poco dinero y cuenten con escasas oportunidades para subsistir y alcanzar estabilización económica y emocional (Nwadiara \& McAdoo, 2000). Ante las muchas experiencias traumáticas que reportan los participantes, que implican reportes de inseguridad y hasta amenaza a la propia vida, el Estatuto de Refugio significa un alivio, pero también involucra enfrentarse a un futuro incierto, ante el cual muchas veces no se cuenta con los medios económicos para hacerle frente.

El estrés por el estatuto de refugio encierra eventos burocráticos tales como hacer los trámites, esperar el resultado de la solicitud, renovar el carné periódicamente y realizar trámites en instituciones públicas, entre otros. El hecho de que estos aspectos representen una dimensión del estrés por aculturación claramente diferenciada, concuerda con resultados previos de investigación en donde se ha observado que uno de los factores que predicen un inadecuado ajuste sociocultural, está vinculado con el hecho de que las personas no cuenten con un buen estatus de inmigración, ya que al no contar con una adecuada situación legal los procesos de adaptación se ven fuertemente afectados (Zlobina et al., 2005).

Los esfuerzos por adaptarse a la sociedad receptora implicaron un menor grado estrés para los entrevistados. Lo anterior puede deberse a que no se perciben grandes diferencias culturales entre Costa Rica y Colombia (ej. marcadas diferencias étnicas, idiomas distintos o lejanía geográfica), por lo que es más probable que esta faceta de aculturación sea, intrínsecamente, menos conflictiva (Berry, 1997; Ye, 2005).

En este estudio no se encontraron variaciones significativas en las dimensiones del estrés según el sexo, la edad u otras variables sociodemográficas de los participantes. Lo anterior difiere de los resultados de investigaciones previas que indican que los hombres tienden a reportar un mayor grado de salud, mayores experiencias de discriminación, menores niveles de estrés por aculturación y menor cantidad de redes de apoyo (Jasinskaja-Lahti et al., 2006). Futuras investigaciones con muestras probabilísticas de mayor tamaño permitirían detectar 
patrones de respuesta diferenciados por sexo y otras variables sociodemográficas relevantes.

En lo que respecta al impacto relativo de la salud, las redes de apoyo y la discriminación, los resultados del presente estudio indican que el principal factor que explica la varianza del estrés posemigración es la percepción de la discriminación. Estos resultados concuerdan claramente con la investigación previa sobre estigmatización social (Major \& O’brien, 2005). En diversos grupos sociales socialmente estigmatizados, la discriminación se ha visto empíricamente vinculada con salud física y mental disminuida (Clark, Anderson, Clark \& Williams, 1999), con insatisfacción personal (Smith, et al., en prensa) con baja autoestima (Schmitt, Branscombe, Kobrynowicz \& Owen, 2002), con un detrimento en el rendimiento académico (Nguyen \& Ryan, 2008) y con mortalidad infantil y pobreza (Behrman, Gaviria \& Székely, 2003). Dado el clima de las relaciones interculturales costarricenses, no es de extrañar, entonces, que las experiencias de discriminación vividas en Costa Rica se perfilen como el mayor factor de riesgo de estrés en los distintos ámbitos del proceso de aculturación posmigratorio.

Para el caso específico del estrés por las demandas de adaptación a la sociedad receptora, los resultados indican que además de la discriminación percibida, los años de residir en el país se constituyen en un predictor importante. Nuestros datos convergen con los resultados de otros estudios que indican que a mayor tiempo de vivir en el país de acogida menor es el nivel de estrés por aculturación (Miranda \& Mathery, 2000). Esto es así, en parte, porque los individuos han tenido menores grados de movilidad (Ward, 2008) y experimentan procesos más avanzados de adaptación psicológica y sociocultural (Zlobina et al., 2005).

Por último, resulta importante recordar que los predictores aquí estudiados no explicaron la variabilidad del estrés por preparación para la salida de Colombia. Evidentemente, estos predictores hacen referencia a estados (salud mental actual), eventos (experiencias de discriminación) y condiciones (redes de apoyo actuales) posmigratorias, no necesariamente vinculadas con el estrés vivido en
Colombia antes de la partida. Diseños de investigación longitudinales, acompañando a las personas refugiadas desde la salida de su país de origen hasta el establecimiento en la sociedad receptora, son necesarios para conocer el impacto de estas variables a lo largo de este complejo proceso.

Las implicaciones de estos resultados para el trabajo con refugiadas son claras. El acompañamiento y asesoría de las personas refugiadas en su país de origen resulta de vital importancia, sobre todo porque en muchas ocasiones las razones de partida están vinculadas a amenazas a la integridad de las personas, causante de estrés postraumático y profundos deterioros de la salud mental. Por otro lado, la rápida inserción al país de origen también resulta fundamental. La agilización de los trámites burocráticos para asegurar estabilidad legal y el desarrollo de mecanismos que aseguren la reunificación familiar, así como la autonomía y autosuficiencia económica, ayudarían a una mejor integración y por ende a una disminución del estrés concomitante. Finalmente, el trabajo en torno a la construcción de una sociedad receptora solidaria y respetuosa de las diferencias culturales, resulta de gran importancia. Las representaciones negativas sobre los refugiados, el trato injusto y, en general, el clima hostil que acompaña muchas veces la "recepción" de las personas refugiadas, representan potenciales fuentes de estrés que, como se observó en este estudio, son el principal obstáculo para la plena inserción de las personas refugiadas en la nueva cultura, limitan el ejercicio de sus derechos y disminuyen su capacidad de adaptación social, cultural y psicológica.

\section{Limitaciones}

En el presente estudio se trabajó con una muestra pequeña de personas refugiadas colombianas, lo cual limita la potencia o sensibilidad del estudio para captar las relaciones entre las variables. Por otra parte, las personas fueron contactadas a través de las agencias nacionales e internacionales encargadas de la protección de personas refugiadas, lo cual limita la generalización de los resultados por autoselección. En otras palabras, estos datos no 
reflejan las experiencias de aquellos y aquellas que ni siquiera cuentan con el apoyo de estas agencias. Por último, este es un estudio correlacional, lo que impide sostener inferencias sobre la relación causal entre las variables estudiadas.

\section{Conclusión}

A pesar de las limitaciones del estudio, este acercamiento a la realidad de las personas refugiadas resulta de gran valor por sus implicaciones prácticas. La principal conclusión que podemos extraer de estos datos, es que la atención a personas refugiadas amerita un trabajo simultáneo con la sociedad de acogida que les permita ejercer plenamente sus derechos.

\section{Referencias}

Allport, G. (1954). The nature of prejudice. Reading, MA: Perseus Book Publishing.

Alto Comisionado de las Naciones Unidas para los Refugiados. (2008). La situación de las personas refugiadas en Costa Rica (Informe de trabajo de la Agencia de las Naciones para los Refugiados en Costa Rica). Recuperado de http://www.acnur.org/ biblioteca/pdf/7002.pdf?view $=1$

Álvaro, J. L., Torregrosa, J. R. \& Garrido, A. (1992). Estructura social y salud mental. En J. L. Álvaro, J. R. Torregrosa \& A. Garrido (Comps.), Influencias sociales y psicológicas de la salud mental (pp. 9-30). Madrid: Siglo XXI Editores.

Behrman, J. R., Gaviria, A. \& Székely, M. (Eds.). (2003). Who's in and who's out: Social exclusion in Latin America. Washington: Inter-American Development Bank.

Berry, J. W. (1997). Immigration, acculturation, and adaptation. Applied Psychology: An International Review, 46, 5-68.

Berry, J. W. (2005). Acculturation: Living successfully in two cultures. International Journal of Intercultural Relations, 29, 697-712.

Berry, J. W. (2008). Globalization and acculturation. International Journal of Intercultural Relations, 32, 328-336.
Berry, J. W. \& Sam, D. (1997). Acculturation and adaptation. In J. W. Berry, M. H. Segall, \& C. Kagitcibasi (Eds.), Handbook of cross-cultural psychology (Vol. 3, pp. 291-326). Boston: Ally and Bacon.

Clark, R., Anderson, N. B., Clark, V. R. \& Williams, D. R. (1999). Racism as a stressor for African Americans: A biopsychosocial model. American Psychology, 54, 805-816.

Graves, T. (1967). Psychological acculturation in a tri-ethnic community. South-Western Journal of Anthropology, 23, 337-350.

Hovey, J. (2000). Psychological predictors of acculturative stress in Mexican immigrants. The Journal of Psychology, 134, 490-502.

Jasinskaja-Lahti, I., Liebkind, K., Jaakkola, M. \& Reuter, A. (2006). Perceived discrimination, social support networks, and psychological well-being among three immigrants groups. Journal of CrossCultural Psychology, 37, 293-311.

Keel, M. \& Drew, N. (2004). The settlement experiences of refugees from the former Yugoslavia: Acculturation, ethnic identity, ethnicity, community and social network development. Community, Work \& Family, 7, 95-115.

Knipscheer, J. \& Kleber, R. (2006). The relative contribution of postraumatic and acculturative stress to subjective mental health. Journal of Clinical Psychology, 3, 341-353.

Liebkind, K. (1996). Acculturation and stress: Vietnamese refugees in Finland. Journal of Cross-Cultural Psychology, 27, 161-180.

Major, B. \& O'brien, L. T. (2005). The social psychology of stigma. Annual Review of Psychology, 56, 393-421.

Miranda, A. \& Mathery, K. (2000). Socio-psychological predictors of acculturative stress among Latino adults. Journal of Mental Health Counseling, 4, 306-317.

Nesdale, A. R., Dharmalingam, S. \& Kerr, G. K. (1987). Effects of subgroup ratio on stereotyping. European Journal of Social Psychology, 17, 353-356.

Nguyen, H. D. \& Ryan, A. M. (2008). Does stereotype threat affect test performance of minorieites and women? A meta-analysis of experimental evidence. Journal of Applied Psychology, 93, 1314-1334. 
Nwadiara, E. \& McAdoo, H. (2000). Acculturative stress among Amerasian refugees: Gender and racial differences. Academia Research Library, 31, 477-488.

Organización Mundial de la Salud. (2004). Promoción de la salud mental. Conceptos. Evidencia emergente. Práctica. (Informe compendiado). Recuperado de http://www.who.int/mental health/evidence/ promocion de la salud mental.pdf

Organización Panamericana de la Salud. (1996). Clasificación Internacional de Enfermedades y Problemas Relacionados con la Salud (CIE-10) (10ª revisión). Washington: Autor.

Schmitt, M. T., Branscombe, N. R., Kobrynowicz, D. \& Owen, S. (2002). Perceiving discrimination against one's gender group has different implications for well-being in women and men. Personality and Social Psychology Bulletin, 28, 197-210.

Smith, V. (2005). Discriminación percibida y autoestima en jóvenes de grupos minoritarios y mayoritarios en Costa Rica. Revista Interamericana de Psicología, 39, 93-106.

Smith, V. (2006). La psicología social de las relaciones intergrupales: modelos e hipótesis. Revista Actualidades en Psicología, 20, 45-71.

Smith, V., Moreno, M., Román, N., Kirschman, D., Acuña, M. \& Víquez, S. (en prensa). Discriminación social, consecuencias psicológicas y estrategias de afrontamiento en miembros de grupos sociales estigmatizados. En I. Dobles \& S. Baltodano (Eds.), Dominación, compromiso y transformación social. San José: Editorial de la Universidad de Costa Rica.
Tajfel, H. \& Turner, J. (1979). An integrative theory of intergroup conflict. In S. Worchel \& W. G. Austin (Eds.), The social psychology of intergroup relations (pp. 33-47). Monterey, CA: Brooks/Cole.

Universidad de Costa Rica \& Agencia de las Naciones Unidas para los Refugiados. (2004). Diagnóstico sobre el grado de integración local de la población refugiada colombiana en Costa Rica, 2004. San José. Costa Rica: Editorama.

Ward, C. (2006). Acculturation, identity and adaptation in dual heritage adolescents. International Journal of Intercultural Relations, 30, 243-259.

Ward, C. (2008). Thinking outside the Berry boxes: New perspectives on identity, acculturation and intercultural relations. International Journal of Intercultural Relations, 32, 105-114.

Ward, C. \& Kennedy, A. (1993). Where's the "culture" in cross-cultural transition: Comparative studies of sojourner adjustment. Journal of Cross-Cultural Psychology, 24, 221-249.

Williams, C. L. \& Berry, J. W. (1991). Primary prevention of acculturative stress among refugees: Application of psychological theory and practice. American Psychologist, 46, 632-641.

Willis, G. (2005). Cognitive Interviewing: A tool for improving questionnaire design. London: Sage.

Ye, J. (2005). Acculturative stress and use of the internet among East Asian international students in the United States. CyberPsychology \& Behavior, 8, 154-161.

Zlobina, A., Basabe, N., Paez, D. \& Furnham, A. (2005). Sociocultural adjustment of immigrants: Universal and group specific predictors. International Journal of Intercultural Relations, 10, 1-17. 


\section{Apéndice}

Medición del estrés por aculturación

\begin{tabular}{|c|c|c|c|c|c|}
\hline ¿Qué tan estresante (tenso o angustiante) le resultó...? & $\begin{array}{c}\text { Nada } \\
\text { estresante }\end{array}$ & & & & $\begin{array}{c}\text { Muy } \\
\text { estresante }\end{array}$ \\
\hline Tomar la decisión de salir de Colombia & 1 & 2 & 3 & 4 & 5 \\
\hline Prepararse para salir de Colombia & 1 & 2 & 3 & 4 & 5 \\
\hline Conseguir el dinero para venirse & 1 & 2 & 3 & 4 & 5 \\
\hline Salir de Colombia & 1 & 2 & 3 & 4 & 5 \\
\hline Trasladarse de Colombia a Costa Rica & 1 & 2 & 3 & 4 & 5 \\
\hline Tener que separarse de su familia & 1 & 2 & 3 & 4 & 5 \\
\hline Dejar de ver a los amigos & 1 & 2 & 3 & 4 & 5 \\
\hline Llegar a Costa Rica & 1 & 2 & 3 & 4 & 5 \\
\hline Hacer los trámites para el refugio & 1 & 2 & 3 & 4 & 5 \\
\hline Esperar el resultado de la solicitud de refugio & 1 & 2 & 3 & 4 & 5 \\
\hline Cubrir sus necesidades básicas (vivienda, alimentación, transporte) & 1 & 2 & 3 & 4 & 5 \\
\hline Encontrar un lugar para vivir & 1 & 2 & 3 & 4 & 5 \\
\hline Encontrar un trabajo & 1 & 2 & 3 & 4 & 5 \\
\hline Estabilizarse económicamente & 1 & 2 & 3 & 4 & 5 \\
\hline Realizar trámites en bancos & 1 & 2 & 3 & 4 & 5 \\
\hline $\begin{array}{l}\text { Realizar trámites en instituciones públicas, por ejemplo: el seguro social, el } \\
\text { instituto de electricidad (ICE), otros }\end{array}$ & 1 & 2 & 3 & 4 & 5 \\
\hline Acceso de los hijos a la educación & 1 & 2 & 3 & 4 & 5 \\
\hline Salir a conocer las ciudades (Ej. paradas de buses) & 1 & 2 & 3 & 4 & 5 \\
\hline Ir de compras & 1 & 2 & 3 & 4 & 5 \\
\hline Adaptarse a las formas de hablar de los costarricenses & 1 & 2 & 3 & 4 & 5 \\
\hline Iniciar el contacto con los vecinos & 1 & 2 & 3 & 4 & 5 \\
\hline Hacer amigos & 1 & 2 & 3 & 4 & 5 \\
\hline Establecer relaciones con las personas con las que trabaja & 1 & 2 & 3 & 4 & 5 \\
\hline Restricciones para viajar a Colombia por ser refugiado & 1 & 2 & 3 & 4 & 5 \\
\hline La supervisión que se hace sobre las personas refugiadas & 1 & 2 & 3 & 4 & 5 \\
\hline Tener que renovar el carné de refugio periódicamente & 1 & 2 & 3 & 4 & 5 \\
\hline Las costumbres costarricenses & 1 & 2 & 3 & 4 & 5 \\
\hline Las comidas costarricenses & 1 & 2 & 3 & 4 & 5 \\
\hline
\end{tabular}


Karla Ugalde-Watson, Vanessa Smith-Castro, Marjorie Moreno-Salas, Jose Miguel Rodríguez-García

\begin{tabular}{lccccc}
\hline \multicolumn{1}{c}{ ¿Qué tan estresante (tenso o angustiante) le resultó...? } & $\begin{array}{c}\text { Nada } \\
\text { estresante }\end{array}$ & & \multicolumn{2}{c}{$\begin{array}{c}\text { Muy } \\
\text { estresante }\end{array}$} \\
\hline Las actitudes de los costarricenses & 1 & 2 & 3 & 4 & 5 \\
La forma de ser del costarricense & 1 & 2 & 3 & 4 & 5 \\
Los comportamientos de los costarricenses & 1 & 2 & 3 & 4 & 5 \\
Los valores costarricenses & 1 & 2 & 3 & 4 & 5 \\
La mentalidad del costarricense & 1 & 2 & 3 & 4 & 5 \\
\hline
\end{tabular}

Fuente: elaboración propia. 